\title{
SPOTur-Tourists Protection and Orientation System in Inhospitable Environments
}

\author{
José Paulo Lousado*, Armando Cruz, Sandra Antunes \\ Escola Superior de Tecnologia e Gestão de Lamego, Instituto Politécnico de Viseu, Lamego, Portugal. \\ * Corresponding author. Tel.: +351 916747920; email: jlousado@estgl.ipv.pt \\ Manuscript submitted September 4, 2018; accepted October 8, 2018. \\ doi: 10.17706/ijcce.2018.7.4.136-144
}

\begin{abstract}
Information and communication technologies have evolved over the last few years in an extraordinary way, especially in the potential of mobile communications, high-speed networks and bandwidth. The present work presents a system model, from the initial design to the prototype, allowing the real time monitoring of tourists in inhospitable environments, namely agricultural regions and mountainous regions of high risk. Real-time tracking incorporates vital-signal monitoring equipment that will allow tour operators to verify in real-time the physical state of their customers - the users of the system. This system intends to make a significant leap in the use of ubiquitous and non-intrusive computing, in support of tourism-related entities operating in regions with some degree of danger, with publics that in turn have some restrictions in terms of mobility, particularly seniors, over 60 years of age or suffering from some pathology that makes mobility difficult for them. This article uses as an application model to the Douro region, that is World Heritage from UNESCO, where morphological and relief features are the ideal place to test the proposed system.
\end{abstract}

Keywords: Orientation systems, support technologies, intelligent systems, pervasive systems.

\section{Introduction}

Currently the information systems oriented to the management and monitoring of individuals, are in a state of immeasurable evolution. Pervasive and ubiquitous computing is present in most home systems, from small devices of regular use, such as a mere blood pressure meter, to larger equipment, such as home appliances, energy efficiency controllers, and more.

The health area is the one that has most benefited from this type of miniaturized technology, often overlooked, with application in the field of vital signs monitoring, in telemedicine, in the use of equipment permanently connected to the Internet, not invasive, that transmits and adjusts its behaviour according to the needs of the user [1].

The Internet of Things, terminology recently used to designate miniaturized systems permanently connected to the network, is currently revolutionizing the way we use technology at the highest level [2].

With the technology currently available, it is possible to combine devices with heterogeneous systems, such as Smartphones with mobile network (3G / 4G and in future to 5G), Bluetooth devices, Wireless networks, sensors, and others, interacting among them, providing operating fully automated adaptive environments, taking advantage of these infrastructures, naturally improving people's quality of life. The advent of the expansion of smart cities is also an excellent example of the use of the Internet of Things, the 
use of Artificial Intelligence, in which ubiquitous computing systems daily collect and generate huge amounts of data, requiring not only Cloud Storage but also its immediate processing, for the advantage of citizens [3]. An example of this is the use of real-time processed data by decision support systems in busy cities, leading buses to choose the most traffic-efficient routes.

Another application of the Internet of Things in the area of mobility, is to condition access to certain sites in terms of levels of pollution, thus preventing certain vehicles from increasing levels of concentration of pollutants, while preventing the exposure of people to environments too contaminated [3], [4].

Using the concepts associated with Smart Cities monitoring, and the use of ubiquitous and pervasive computing in people's quality of life, we developed a concept and an application model in the field of tourist monitoring in regions of high slope, rough terrain, and for people with reduced mobility, such as the elderly population.

The Douro region is visited annually by thousands of tourists, mainly senior tourists. Based on this reality, accompanied by the will of the tourists who visit the Douro region, also on pedestrian routes, we present in this article, a tourist protection and orientation system called SPOTur.

\section{System Modelling}

The study and application of certain types of portable easy operation sensors have grown considerably. Portable sensors, namely the accelerometer, of small size, low power consumption and high precision, have been used in many tests in individuals who have some types of diseases that limit their mobility, allowing validation in real time if a particular individual suffers an abrupt fall [5]. Several authors have worked with these and other sensors, showing the advantage of using these small devices to aid and monitor people.

In [6] the use of accelerometers showed that it is possible to determine the cause of a fall through Machine Learning algorithms, exploring a previously unfinished strand mainly in the field of adult monitoring.

More recently, in [7], [8], it can be verified that the portable sensors have enormous potentialities in the field of monitoring people's movements and in the analysis of incidence of falls.

Considering that currently mobile communication devices, commonly known as Smartphones, have several sensors incorporated, including accelerometer, GPS, etc., we propose in the present work, to explore and enhance the use of these sensors, to real-time monitor tourists who move in risky natural areas, naturally propitious to eventual falls, monitoring not only their location, but also their ability to move, their pulse, travel path and fatigue levels.

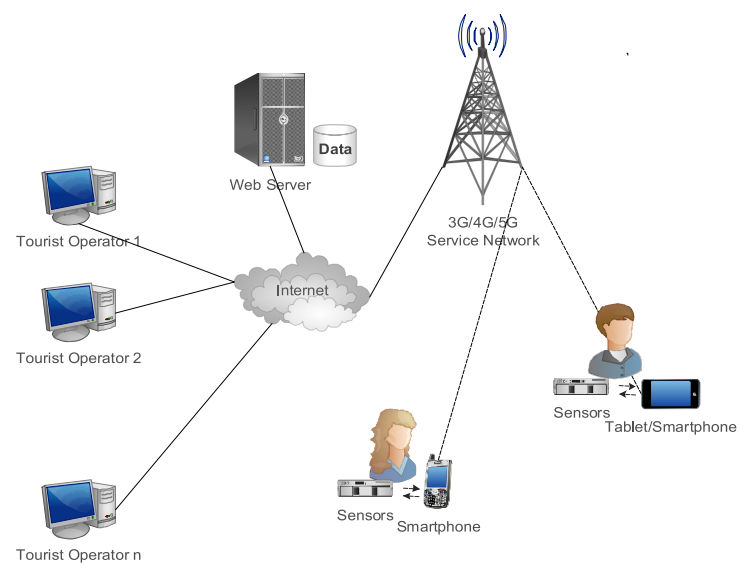

Fig. 1. System architecture.

The system consists of an application developed for mobile devices (Smartphone), with access to the 
Internet (3G / 4G), which will access a web server, interconnected by Bluetooth to a microcontroller that interconnects in addition to the sensors already mentioned, body temperature sensors [9], humidity [10] and pulse rate [11]. The communication is carried out via Web Services, sending in real time the data results, of monitoring the users, to a server that will proceed to its storage in database, as showed in Fig. 1.

\subsection{Functional Requirements}

At the level of functional requirements, the following operations are considered as most relevant:

- Secure connection service with registration, authentication, and user validation;

- Differentiation of available services for different user types - Administrator, Client (tour operator), User, Guest (non-authenticated user);

- Data collection of the coupled sensors (accelerometer, temperature, pulse, humidity);

- Geo-referencing data collection (GPS);

- Ability to send user's data by Web services;

- Parameter set and configuration of the application installed on mobile devices (remotely);

- Real-time analysis of the data collected (local processing) for detecting deviations beyond a certain allowed range;

- Notifications to the local device;

- Alert of abnormal occurrence to the operator (local);

- Real-time representation of the geographical location of mobile users.

The main use cases of the System are shown below, in Fig. 2, representing the three main types of users and the use cases associated with them.

All users need to log in before performing any task on the system.

Production User has access to all system management, including user management and registered path management.

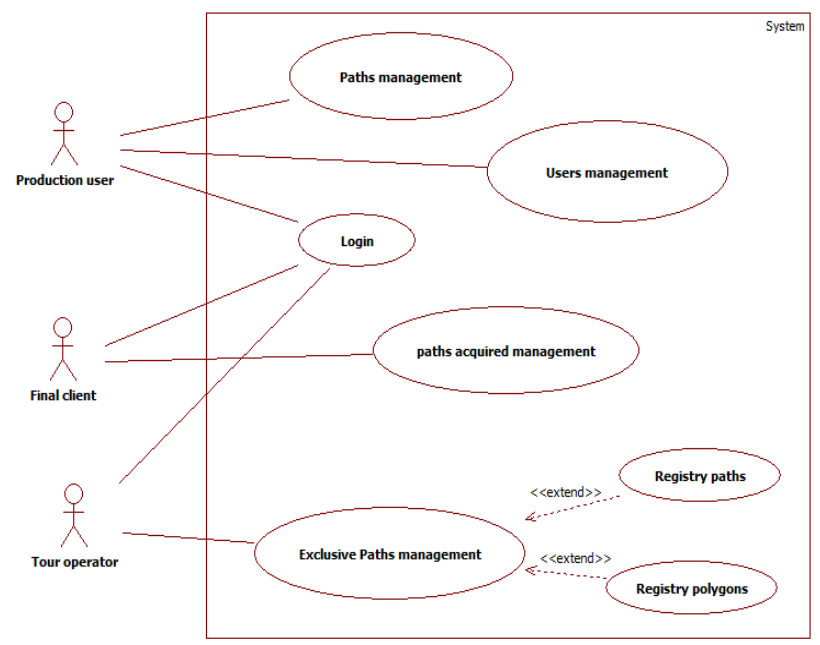

Fig. 2. Main use case model.

The Tour Operator has exclusive access to its routes and the routes that are acquired, thus allowing them to be monitored as soon as they are acquired.

The Final Client has access to the existing paths, allowing them to acquire and navigate them.

\subsection{Non-functional Requirements}

No less important are the non-functional requirements, which are responsible for guaranteeing functionality and operability in accordance with minimum quality standards, namely at the level of: 
- Reliability - the system must be tested for its robustness, guaranteeing its operation in extreme situations, namely: a) low network signal, b) influence of weather conditions, c) ability for local storage of data in the absence of a network.

- Safety - the system must be safe from the user's point of view, namely placing the sensors in places that do not compromise the user's mobility, and strong enough so it won't easily deteriorates making the data gathering useless.

- Usability - should be user-friendly, and multilingual. At this level it is intended that the system has an interface based on options, triggered by an interface that allows individuals with reduced mobility or other physical limitations, but not a visual one, to operate the equipment without difficulty.

In order to ensure the functionality of the system, the integration and operational tests will be extremely important, estimating an effort placed between $30 \%$ and $40 \%$ of the total effort required by the development team.

\subsection{Conceptual Scheme}

The application support suite includes several modules, namely:

- Data Collection Module;

- Base Web Services Module (Core Server Core)

- Data Submission and Processing Module;

- Graphical representation of the location of individuals;

- Module for Monitoring and Control of Routes;

- Configuration and Parameterization module;

- User Management Module.

\subsection{Relational Database}

The database is quite simple, having been normalized according to the relational model, being constituted by the following entities and relations (Fig. 3.):

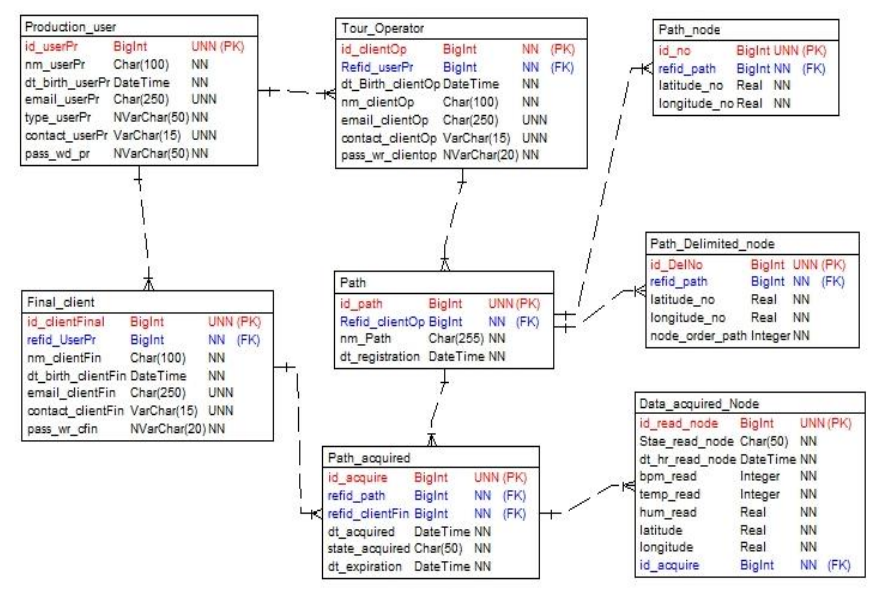

Fig. 3. Database scheme.

Production_user - represents the system administrator user, responsible for the management of operational client data (Tour_operator) and final clients (Final_client).

Tour_Operator - represents the operational client, who is a tour operator. It is responsible for the creation of pedestrian routes and for the registration of the nodes allowed on the routes, defining the safety area of the route. 
Path_node - allows to register the data of each node, forming the footpath.

Path_Delimited_node - allows to register the data of each node, which create the security polygon.

Final_client - represents the end customer (tourist) who uses the system and purchases the route with operational client monitoring (Path_acquired).

Path_acquired - allows storing the data related to the pedestrian paths acquired by the client, which will be monitored and monitored.

Data_acquired_node - allows the recording of each moment of data collection with the geo-referencing information and vital signs of the user.

\section{Interface}

The System Interface is focused on the main functionalities and should present the information according to the status and type of user. The Fig. 4 shows the interface to the System Administrator's Backoffice system, where it can control the activity of the clients and the management of the various users of the system.

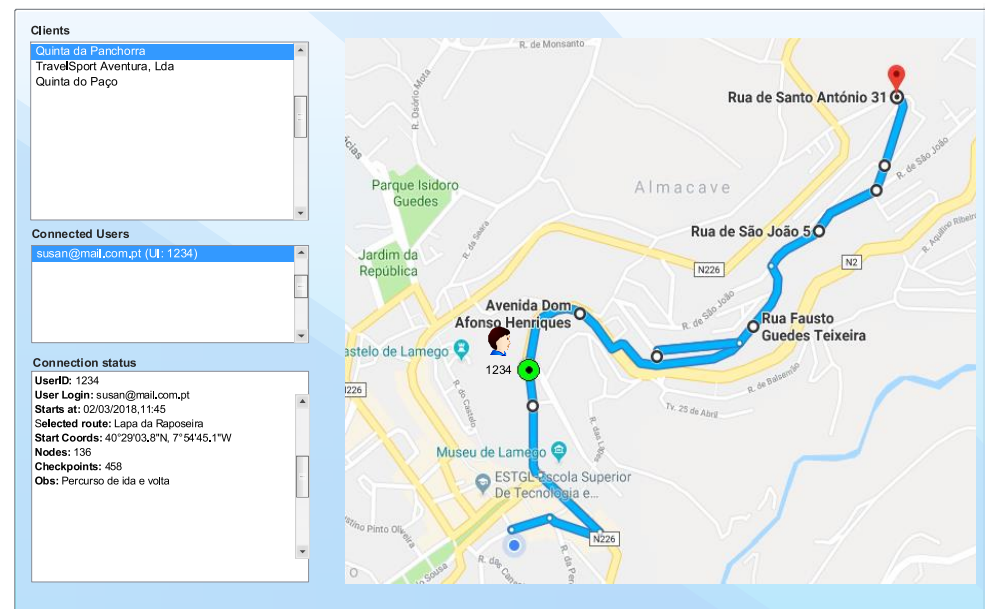

Fig. 4. Monitoring interface (conceptual backoffice model).

\subsection{Proof of Concept}

From business customers - tour operators - they will have at their disposal an interface that will allow them to monitor the execution of the routes by the users of the system (second level of customer). They will have access to their customers' information, such as the route they follow, waiting times, detours, notices sent, and vital signs monitoring.

The interface will be similar to the one of the General System Administrator, with the particularity of having access only to paths, and to registered users for those paths, within a limited time period.

The user will have a mobile application that able to access the web services of the system, which will send the geo-referencing signals and vital signs collected by the sensors incorporated by GSM. Whenever the mobile network signal does not allow data to be sent, it will be stored in a temporary local database, which will be synchronized with the server as soon as the network signal is received again.

The system will monitor users in such a way that, in case the waiting time for data reception is exceeded, plus a tolerance set by the Tour Operator (Business Client), a search is triggered and eventually, the search engines are activated to the nearest location (last contact point collected).

Fig. 5, shows the simulator to operate in real time, allowing to verify in the laboratory how the system responds to variations in the location of the individuals being monitored. 
It allows to verify that every time that an individual (tourist) leaves the region of security, it immediately appears in red the signalling that something dangerous is happening.

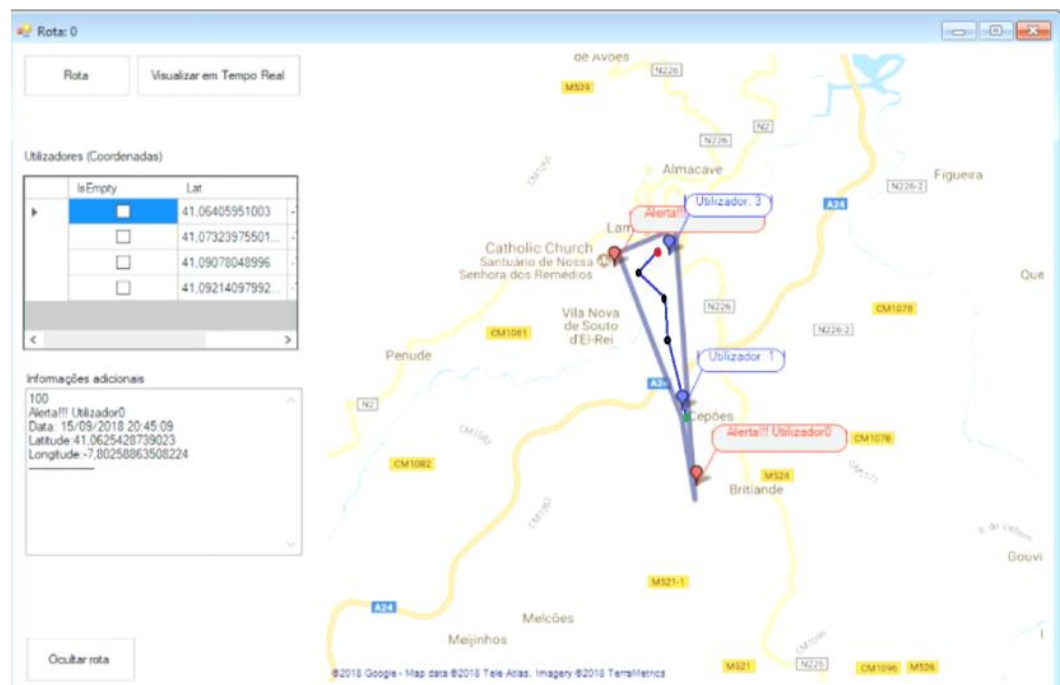

Fig. 5. Real time monitoring interface (sample of backoffice application).

\subsection{Application Scenario}

Considering the Douro as beautiful as it is dangerous, this region recognized with World Heritage by UNESCO, is the scenario of ideal application. It has several pedestrian routes between wineries, with steep slopes in some places, with irregular mobile network signal coverage.

The clients of the tour operators are traditionally seniors, aged between 50 and 65 years, who enjoy walking outdoors, nature tourism and wine tourism, where about $60 \%$ of these are foreigners or residing abroad from Portugal. This will be the target audience for the system and the particular application scenario.

The monitoring equipment includes several sensors (temperature, humidity and heart rate) interconnected and connected to a computer system based on the microcontroller Arduino UNO that can be connected by Bluetooth to the customer's Smartphone.

Alternatively use another device based on Raspberry PI 3 that will allow the user a periodic monitoring between the point of departure and the expected point of arrival.

The user can move freely between the starting and finishing points, conditioned by the passage through certain nodes, which usually coincide with nodes of divergence - alternative routes, routes to avoid or routes with no exit.

Whenever the user moves beyond the allowable tolerance, the system will issue an alert to the control panel, informing that if there are deviations, the operator can choose to send warnings to the user, by written message, SMS or audio signal.

If the system continues to check the deviations without obtaining a response from the user, or in the case of a loss of the signal, other users of the system that are part of the same group may be contacted, or if there is no response, the procedure of search.

The confirmation of the passage through the main nodes is automatically calculated when the system sends two consecutive data collection points, where the coordinates of the main node are among the same, within the parameters allowed in terms of tolerance.

It is essential to gauge the relationship between the actual location of the user and a reference point of the course, thus validating if it is within the allowed tolerance defined by the delimiter polygon. 


\subsection{Mobile Access}

Mobile access to the system is done via web services, where we develop an application that uses these services. Using these services, from the moment the user loads the application and starts a route, it will automatically appear on the tour operator's monitor, displaying the vital and geo-referencing data.

The map with the route and its safety polygon will appear on the Smartphone, as well as the data collected on the sensors attached to the specially prepared wristband, like we can see in Fig. 6.
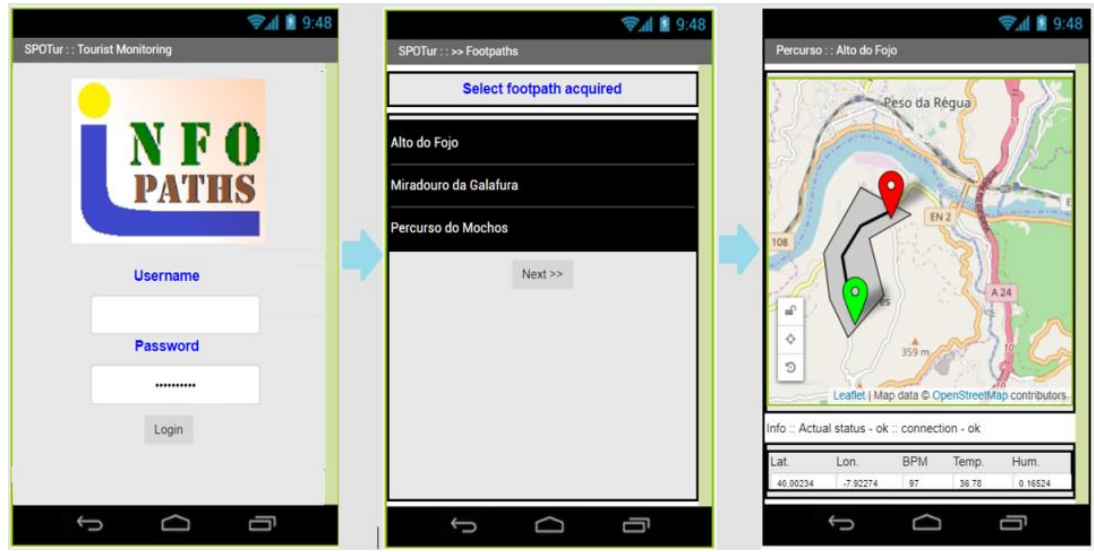

Fig. 6. Prototype of mobile interface.

At each collection time, every 3 seconds by default (can be parameterized to another value), the data is collected and sent automatically to the server, where the Tour Operator follows the movement.

\section{Web Service API}

To support application and future applications development, we have been developing a set of web services that constitute the API for potential application designers and developers (Fig. 7.).

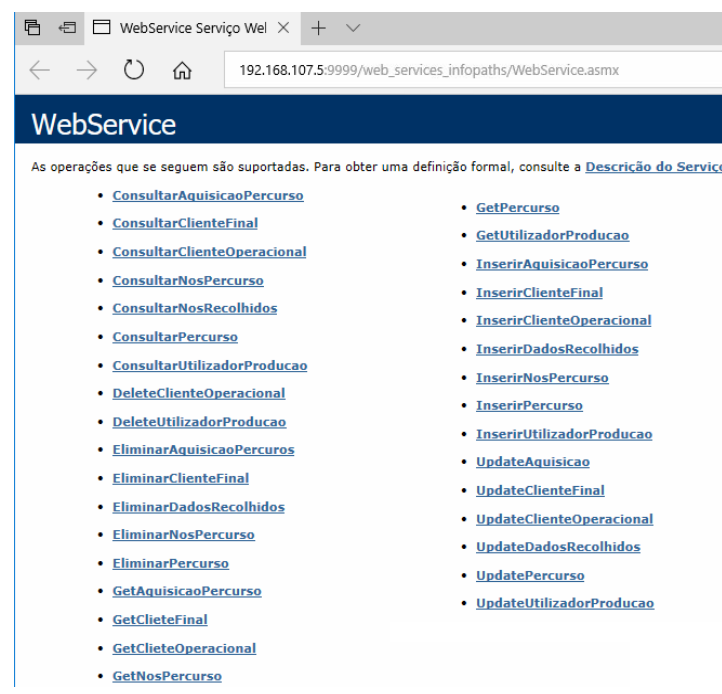

Fig. 7. Web services for API support.

These web services allow users to perform all the tasks that the system needs, namely queries, data insertion, change and deletion of data.

For each set of web services, there is user authorization control, preventing unauthorized users from performing tasks on the system for which they are not authorized. 


\section{Conclusion}

The present project includes a simulator that will allow to verify in a controlled environment the effectiveness of the system, also allowing the system to be tested on routes with real coordinates without the need to travel immediately to the terrain.

This work is still in the development and component testing phase, but it has enormous potential in monitoring and accompanying tourists, mainly seniors, who travel on pedestrian routes with a high risk of fall or other negative events.

Traditionally in regions such as the Douro World Heritage, tourists feel a great desire to walk outdoors, enjoying the air quality and all the environmental surroundings and natural beauty.

However, regions with steep areas and terraces are prone to accidents and, therefore, tourists' monitoring by tour operators helps to guarantee that in the event of any risk to happen, the means of distress can be activated immediately.

Although the proposed system is not yet fully developed, its potentiality and usefulness in the field of the monitoring of people with difficulty of locomotion can be perceived.

The concept and technologies developed for this article can be easily adapted to other contexts, particularly in the social field, where monitoring of people with psychological illnesses suffering from diseases such as Alzheimer's disease, dementia or other similar pathologies may prevent disappearance of these people.

Finally, with the new data protection regulation approved by European Directive 2016/679 [12], of the European Parliament and of the Council of 27 April 2016, the collection of personal data should scrupulously comply with the Directive and the respective data collection authority must be requested for statistical and scientific purposes to the National Data Protection Commission.

\section{Acknowledgment}

This article is a result of the project IFO PATHS, Reference code: NORTE-01-0145-FEDER-023623, supported by Norte's Operational Programme (NORTE 2020), under the PORTUGAL 2020 Partnership Agreement, through the European Regional Development Fund (ERDF) and FCT.

\section{References}

[1] Wickramasinghe, N. (2013). Pervasive computing and healthcare. In R. K. Bali, et al. (Eds.), Pervasive Health Knowledge Management. New York.

[2] Chin, W. S., Kim, H. S., Heo, Y. J., \& Jang, J. W. (2015). A context-based future network infrastructure for IoT services. Procedia Comput. Sci., 56(1), 266-270.

[3] Skouby, K. E., \& Lynggaard, P. (2014). Smart home and smart city solutions enabled by 5G, IoT, AAI and CoT services. Proceedings of the 2014 International Conference on Contemporary Computing and Informatics (IC3I) (pp. 874-878).

[4] Fazio, M., Celesti, A., Puliafito, A., \& Villari, M. (2015, Jan.). Big data storage in the cloud for smart environment monitoring. Procedia Comput. Sci., 52, 500-506.

[5] Ermes, M., Pärkkä, J., Mäntyjärvi, J., \& Korhonen, I. (2008). Detection of daily activities and sports with wearable sensors in controlled and uncontrolled conditions. IEEE Trans. Inf. Technol. Biomed., 12(1), 20-26.

[6] Aziz, O., \& Robinovitch, S. (2009). An analysis of the accuracy of wearable sensors for distinguishing the cause of falls. IEEE Trans. Neural Syst. Rehabil. Eng., 19(6), 670-676.

[7] Shany, T., Redmond, S. J., Narayanan, M. R., \& Lovell, N. H. (2012). Sensors-based wearable systems for monitoring of human movement and falls. IEEE Sens. J., 12(3), 658-670. 
[8] Cruz, A., \& Lousado, J. P. (2018). A survey on wearable health monitoring systems. Proceedings of the CISTI - Conferência Ibérica em Sistemas e Tecnologias de Informação.

[9] Texas Instruments. Temperature Sensor LM35. Retrieved from the website: http://www.ti.com/product/lm35?qgpn=lm35

[10] Ali, A. S., Zanzinger, Z., Debose, D., \& Stephens, B. (2016). Open source building science sensors (OSBSS): A low-cost Arduino-based platform for long-term indoor environmental data collection. Build. Environ., 100, 114-126.

[11] Murphy, J., \& Gitman, Y. (2011). Pulse sensor amped. Retrieved from the website: https://pulsesensor.com/products/pulse-sensor-amped

[12] European Union. (2014, March). Regulation 2016/679 of the European parliament and the Council of the European Union. Off. J. Eur. Communities, 2014, 1-88.

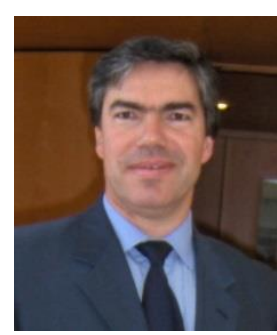

José Paulo Lousado is a researcher at Instituto Politécnico de Viseu, Portugal. He received his PhD in informatics engineering from DETI / University of Aveiro, and master's degree in science, technology and innovation management from DEGEI / University of Aveiro. Has a bachelor in mathematics / informatics from the University of Beira Interior - Covilhã. Since 2003, he is an adjunct professor at the Superior School of Technology and Management of Lamego (ESTGL), Instituto Superior Politécnico de Viseu, Portugal, where he currently teaches curricular units of analysis and design of systems, introduction to programming, management of software projects, engineering project management, database management systems, database systems and multimedia techniques in tourism. He was a member of the research team and is project leader for project NORTE-01-0145-FEDER-023623 (funded by the European Union). His areas of interest are programming, systems analysis, ubiquitous and pervasive systems, bioinformatics and e-learning.

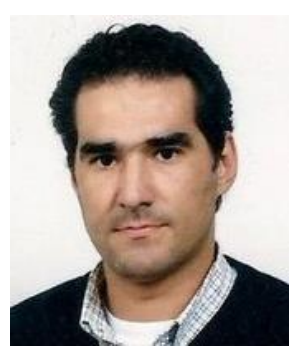

Armando Cruz is a researcher at Instituto Politécnico de Viseu, Portugal. He received his PhD in informatics from UTAD, Trás-os-Montes e Alto Douro University, Vila Real. He was a member of the research team for project NORTE-01-0145-FEDER-023623 (funded by the European Union). His areas of interest are programming, systems analysis, electronic, telecommunications, data mining and machine learning.

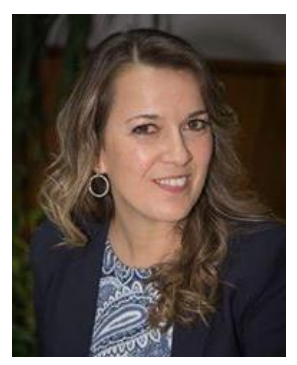

Sandra Antunes is a researcher at Instituto Politécnico de Viseu, Portugal. She received her PhD in educational sciences from Facultad de Educación, Universidad de Salamanca, Salamanca. She has a bachelor degree in sociology from Universidade Nova de Lisboa and accomplished postgraduate studies in sociology of development and social transformation at Faculdade de Economia, Universidade de Coimbra. She is an adjunct professor, since 2014, at Superior School of Technology and Management of Lamego (ESTGL), Instituto Politécnico de Viseu, Portugal, currently teaches subjects as sociology of family, sociocultural anthropology, scientific research methodologies and techniques, and data analysis. She was a member of the research team for project NORTE-01-0145-FEDER-023623 (funded by the European Union). Her research areas fall into demographic and social transformations, educational politics and qualitative data analysis. 